\title{
The anomalous diffusion of polymers in random media
}

\author{
Arti Dua and Binny J. Cherayil \\ Department of Inorganic and Physical Chemistry, Indian Institute of Science, Bangalore 560012, India
}

\begin{abstract}
We re-examine the problem of the diffusion of a Gaussian chain in a fixed array of obstacles using the projection operator formalism introduced by Loring [J. Chem. Phys. 88, 6631 (1988)]. We show that in the limit of long wavelengths, the frequency-dependent monomer friction coefficient that is used in the calculation of the mean square displacement of the center of mass can be rewritten exactly in terms of the time correlation function of the total force on the chain. When the decay profile of the force correlation function is assumed to be exponential, and its dependence on the density of obstacles written in an approximate resummed form, the dynamics of the center of mass is found to be diffusive at long and short times, and subdiffusive (anomalous) at intermediate times. Moreover, the diffusion coefficient $D$ that describes the long-time behavior of the chain at high concentrations of small obstacles is found to vary with chain length $N$ as $N^{-2}$, which is in qualitative agreement with the predictions of the reptation model. These results are obtained in the absence of any mechanism that might incorporate the notion of reptation directly into the calculations, in contrast to Loring's approach, which treats the monomer friction coefficient approximately using a decoupling of segmental motion into parallel and perpendicular components.
\end{abstract}

\section{INTRODUCTION}

Many of the dynamical properties of polymer melts can be traced to the topological constraints that prevent one chain from moving though another. The constraints have a sufficiently complex many-body structure that in models of the melt their effects are often represented by the action of a confining tube on the dynamics of a single chain. The tube itself is sometimes further reduced to a set of fixed interaction sites selected at random from points that lie along its surface. This set of random scattering centers can be regarded as amongst the simplest realizations of the microscopic entanglements that exist in a melt. ${ }^{1,2}$ There seems to be general agreement that a polymer in such an array of scatterers would have to move by sliding down its own contour, in much the same way that defects travel down the length of a rope. Segmental motion of this kind, which is biased along directions parallel to the backbone of the chain, is referred to as reptation. In the polymer/scatterer system, a transition from free to reptative motion is thought to occur at a certain concentration of scatterers, just as in the melt an analogous transition is thought to occur at a certain chain length of the polymers. ${ }^{2-6}$

An especially interesting analytical illustration of this transition was provided by Loring some years ago. ${ }^{6}$ Starting from a generalized Fokker-Planck equation for the dynamics of a Gaussian chain in an array of fixed impenetrable obstacles, Loring showed, using projection operator methods and a variant of a closure relation applied previously to localization problems, that the motion of the chain, if decomposed into transverse and longitudinal components, would exhibit the same long-time dynamical features as the motion of a chain undergoing reptation. The transition to reptation dynamics was preceded by a period of anomalous diffusion, in which the center-of-mass displacement varied sublinearly with time. These results were taken as evidence of the progressive decrease of segmental motion perpendicular to the chain contour as the amount of disorder in the medium increased. The results were also in general agreement with the results of simulations, ${ }^{7-9}$ and could therefore be seen as providing a rigorous microscopic basis for the idea of reptation.

However, a model that at the outset introduces into the chain dynamics a clear difference between parallel and perpendicular modes of segmental motion, would already seem to have predisposed the chain to diffuse reptatively. That such a model largely succeeds in generating results consistent with reptation could well be a reflection of this initial directional bias. The model's utility as a microscopic description of chain dynamics in random media would therefore be more stringently tested if this bias were eliminated. In the present work, we show that this may be done (although not without introducing other approximations).

The following section briefly recapitulates the principal ingredients of Loring's projection operator description of the center-of-mass displacement of a Gaussian chain in a random array of obstacles. We show there that in the long wavelength limit, the calculation of this quantity can be reduced to the evaluation of the time correlation function of the total force on the chain. We then show that a simple ansatz for this correlation function, together with a resummation approximation, lead to an analytic equation for the center-ofmass displacement that shows anomalies that are very similar to those seen in Loring's calculations. Finally, we discuss the implications of these results.

\section{CENTER-OF-MASS MOTION AND THE GENERALIZED DIFFUSION COEFFICIENT}

As indicated, the quantity we shall be interested in is $\left\langle\left\langle\mathbf{r}_{\mathrm{c} . \mathrm{m} .}^{2}(t)\right\rangle\right\rangle$, the mean square displacement of the center of 
mass of a Gaussian chain in time $t$ as a function of the concentration of obstacles in the medium. The double brackets here refer to an average with respect to both the initial equilibrium distribution of the chain, $P_{0}$, and the distribution of the obstacles, $P_{s}$. In the long wavelength limit, $\left\langle\left\langle\mathbf{r}_{\mathrm{c} . \mathrm{m} .}^{2}(t)\right\rangle\right\rangle$ is calculated from a generalized time dependent diffusion constant $D(t)$ according to ${ }^{6}$

$$
\left\langle\left\langle\mathbf{r}_{\mathrm{c} . \mathrm{m} .}^{2}(t)\right\rangle\right\rangle=6 \mathcal{L}^{-1} \frac{\widetilde{D}(s)}{s^{2}},
$$

where $\widetilde{D}(s)$ is the Laplace transform of $D(t), \mathcal{L}^{-1}$ denotes the operation of taking the inverse Laplace transform, and $s$ is the Laplace variable conjugate to $t$. Following Loring, we relate $\widetilde{D}(s)$ to the microscopic dynamics of the polymer/ obstacle system through

$$
\widetilde{D}(s)=\frac{1}{3}\left\langle\left\langle\mathbf{v}_{\text {c.m. }} \cdot\left|(s-L)^{-1}\right| \mathbf{v}_{\text {c.m. }}\right\rangle\right\rangle,
$$

where $\mathbf{v}_{\text {c.m. }}$, the center-of-mass velocity of the chain, is given by

$$
\mathbf{v}_{\mathrm{c} . \mathrm{m} .}=\frac{1}{m N} \sum_{i=1}^{N} \mathbf{p}_{i}
$$

Here, $m$ is the monomer mass, $N$ is the number of monomers, $\mathbf{p}_{i}$ is the momentum of the $i$ th monomer, and $L$ is the following Fokker-Planck operator. ${ }^{6,10}$

$L=\sum_{i=1}^{N}\left[-\frac{1}{m} \mathbf{p}_{i} \cdot \boldsymbol{\nabla}_{\mathbf{r}_{i}}-\mathbf{F}_{i} \cdot \boldsymbol{\nabla}_{\mathbf{r}_{i}}+m \gamma_{\infty}\left(k_{B} T \nabla_{\mathbf{p}_{i}}^{2}+\frac{1}{m} \boldsymbol{\nabla}_{\mathbf{p}_{i}} \cdot \mathbf{p}_{i}\right)\right]$

with $\mathbf{F}_{i}$ the force on the $i$ th monomer, and $\gamma_{\infty}$ the monomer friction coefficient.

In order to obtain an expression for $\widetilde{D}(s)$ that can be suitably approximated, the identity $(X-Y)^{-1}=X^{-1}$ $+X^{-1} Y(X-Y)^{-1}$ is applied to the resolvent operator $(s$ $-L)^{-1}$ so as to rewrite it in the form

$$
\frac{1}{s-L}=\frac{1}{s-L(P+Q)}=\frac{1}{s-L Q}+\frac{1}{s-L Q} L P \frac{1}{s-L},
$$

where $P$ and $Q$ are projection operators that satisfy $P+Q$ $=1$. $P$ is chosen to be

$$
\left.P=\left|\mathbf{v}_{\mathrm{c} . \mathrm{m} .}\right\rangle\right\rangle \frac{m N}{3 k_{B} T}\left\langle\left\langle\mathbf{v}_{\mathrm{c} . \mathrm{m} .} \cdot\right|\right.
$$

whence it follows that $\left.Q\left|\mathbf{v}_{\text {c.m. }}\right\rangle\right\rangle=0$. These results, when substituted into Eq. (2), lead to the following expression for $\widetilde{D}(s)$ :

$$
\begin{aligned}
\widetilde{D}(s)= & \frac{1}{3 s}\left\langle\left\langle\mathbf{v}_{\mathrm{c} . \mathrm{m} .} \cdot \mid \mathbf{v}_{\mathrm{c} . \mathrm{m} .}\right\rangle\right\rangle+\left\langle\left\langle\mathbf{v}_{\mathrm{c} . \mathrm{m} .} \cdot\left|(s-L Q)^{-1} L\right| \mathbf{v}_{\mathrm{c} . \mathrm{m} .}\right\rangle\right\rangle \\
& \times \frac{m N}{3 k_{B} T} \widetilde{D}(s) .
\end{aligned}
$$

On the right-hand side of this equation, the operator $L$ in the expression $\left.L\left|\mathbf{v}_{\text {c.m. }}\right\rangle\right\rangle$ acts both on the dynamical variable $\mathbf{v}_{\text {c.m. }}$. as well as on the equilibrium distribution $P_{0}$. It proves convenient, therefore, to define an operator $L^{+}$such that for any variable $A$,

$$
L P_{0} A=-P_{0} L^{+} A .
$$

For this relation to hold, $L^{+}$must be given by

$$
\begin{aligned}
L^{+}= & \sum_{i=1}^{N}\left[\frac{1}{m} \mathbf{p}_{i} \cdot \nabla_{\mathbf{r}_{i}}+\mathbf{F}_{i} \cdot \boldsymbol{\nabla}_{\mathbf{p}_{i}}\right. \\
& \left.+m \gamma_{\infty}\left(-k_{B} T \nabla_{\mathbf{p}_{i}}^{2}+\frac{1}{m} \mathbf{p}_{i} \cdot \boldsymbol{\nabla}_{\mathbf{p}_{i}}\right)\right] .
\end{aligned}
$$

It then follows that

$$
L \mathbf{v}_{\text {c.m. }} P_{0}=-P_{0}\left(\frac{1}{m N} \mathbf{F}+\gamma_{\infty} \mathbf{v}_{\text {c.m. }}\right),
$$

where $\mathbf{F}\left(=\sum_{i=1}^{N} \mathbf{F}_{i}\right)$ is the total force acting on the chain. Substituting Eqs. (8) and (9) into (7) and using the result $\left\langle\left\langle\mathbf{v}_{\text {c.m. }} \cdot \mid \mathbf{v}_{\text {c.m. }}\right\rangle\right\rangle=3 k_{B} T / m N$, one now finds that $\widetilde{D}(s)$ can be reduced to

$\widetilde{D}(s)=\frac{k_{B} T}{m N} /\left(s+\gamma_{\infty}+\frac{s}{3 k_{B} T}\left\langle\left\langle\mathbf{v}_{\mathrm{c} . \mathrm{m} .} \cdot\left|(s-L Q)^{-1}\right| \mathbf{F}\right\rangle\right\rangle\right)$.

This expression is reduced further as follows: First, $\left\langle\left\langle\mathbf{v}_{\mathrm{c} . \mathrm{m}}\right.\right.$. $\left.\left.\cdot\left|(s-L Q)^{-1}\right| \mathbf{F}\right\rangle\right\rangle$ is written as $s^{-1}\left\langle\left\langle\mathbf{v}_{\mathrm{c} . \mathrm{m} .} \cdot\right| L Q(s\right.$ $\left.-L Q)^{-1}|\mathbf{F}\rangle\right\rangle$ using $(X-Y)^{-1}=X^{-1}+X^{-1} Y(X-Y)^{-1}$ and the identity $\left\langle\left\langle\mathbf{v}_{\text {c.m. }} \cdot \mid \mathbf{F}\right\rangle\right\rangle=0$. Then the average $\langle\langle A|L| B\rangle\rangle$ $\equiv \int d\{\mathbf{x}\} A\left(L B P_{0}\right)$, where $A$ and $B$ are any two dynamical variables and $\{\mathbf{x}\}$ are the set of phase space variables of the chain, is written in the form $\int d\{\mathbf{x}\}(M A) B P_{0}$, where $M$ is an operator that may be shown to be

$M=\sum_{i=1}^{N}\left[\frac{1}{m} \mathbf{p}_{i} \cdot \boldsymbol{\nabla}_{\mathbf{r}_{i}}+\mathbf{F}_{i} \cdot \boldsymbol{\nabla}_{\mathbf{p}_{i}}+m \gamma_{\infty}\left(k_{B} T \nabla_{\mathbf{p}_{i}}^{2}-\frac{1}{m} \mathbf{p}_{i} \cdot \boldsymbol{\nabla}_{\mathbf{p}_{i}}\right)\right]$.

The action of $M$ on $A$ is easily determined when $A$ is the variable $\mathbf{v}_{\text {c.m. }}$. The preceding results, in combination with the Hermitian character of the projectors $P$ and $Q$, can be used to show that

$$
\left\langle\left\langle\mathbf{v}_{\mathrm{c} . \mathrm{m} .} \cdot\left|(s-L Q)^{-1}\right| \mathbf{F}\right\rangle\right\rangle=\frac{1}{m N s}\left\langle\left\langle\mathbf{F}\left|(s-L Q)^{-1}\right| \mathbf{F}\right\rangle\right\rangle
$$

which, after substitution in Eq. (11), leads to

$$
\widetilde{D}(s)=D_{R} /\left(1+s / \gamma_{\infty}+\widetilde{\gamma}(s) / \gamma_{\infty}\right),
$$

where $D_{R}=k_{B} T / m N \gamma_{\infty}$ and

$$
\widetilde{\gamma}(s)=\frac{1}{3 m N k_{B} T}\left\langle\left\langle\mathbf{F} \cdot\left|(s-L Q)^{-1}\right| \mathbf{F}\right\rangle\right\rangle .
$$

The variable $\tilde{\gamma}(s)$ can be interpreted as a generalized frequency dependent friction coefficient. Equation (14) is identical to Eq. (2.17) of Ref. 6, except that the friction coefficient there is defined with a minus sign.

\section{ELIMINATION OF THE PROJECTED DYNAMICS IN $\tilde{D}(s)$}

Equation (14) is the starting point of Loring's analysis of center-of-mass diffusion. The key element in this analysis is an ansatz that provides a resummed expression for the short 
time expansion of $\widetilde{\gamma}(s)$. When segmental motion is decomposed into parallel and perpendicular components, the resummed form of $\widetilde{\gamma}(s)$ leads eventually to a self-consistent equation for the frequency-dependent self-diffusion coefficient in the transverse direction that can be solved analytically. The time dependence of the mean square displacement of the center-of-mass predicted by this expression (as depicted in Fig. 1 of Ref. 6) is characteristically anomalous at intermediate times for high obstacle concentrations. The result can be interpreted as pointing to a reptationlike transition when the concentration of obstacles assumes a critical value. However, the above decomposition of segmental motion into distinct modes along parallel and perpendicular directions is somewhat arbitrary, so it is not entirely clear if the observed transition is not simply a manifestation of this operation.

As we shall now try to show, similar anomalies in center-of-mass diffusion can be seen in the absence of any assumptions about preferred modes of motion. The present analysis also takes Eq. (14) as its point of departure, but thereafter it proceeds by writing $\widetilde{D}(s)$ exactly in terms of a time correlation function that does not involve the projection operators $P$ or $Q$. Two key assumptions are then made, one specifying the nature of the time dependence of this time correlation function, and the other specifying a resummed form for the concentration dependence of the mean square center-of-mass displacement.

We begin by using the relation $Q=1-P$ in Eq. (15), along with the definition of $P$ given in Eq. (6) and the identity used earlier to treat operators in resolvent form. This leads to

$$
\begin{aligned}
\langle\langle\mathbf{F} \cdot| & \left.(s-L Q)^{-1}|\mathbf{F}\rangle\right\rangle \\
= & \left\langle\left\langle\mathbf{F} \cdot\left|(s-L)^{-1}\right| \mathbf{F}\right\rangle\right\rangle-\left\langle\left\langle\mathbf{F} \cdot\left|(s-L)^{-1} L\right| \mathbf{v}_{\text {c.m. }}\right\rangle\right\rangle \\
& \times \frac{m N}{3 k_{B} T}\left\langle\left\langle\mathbf{v}_{\text {c.m. }} \cdot\left|(s-L Q)^{-1}\right| \mathbf{F}\right\rangle\right\rangle .
\end{aligned}
$$

In the second term on the right-hand side of this expression, the first correlation function is written as

$$
\begin{aligned}
& \left\langle\left\langle\mathbf{F} \cdot\left|(s-L)^{-1} L\right| \mathbf{v}_{\text {c.m. }}\right\rangle\right\rangle \\
& \quad=-\frac{1}{m N}\left\langle\left\langle\mathbf{F} \cdot\left|(s-L)^{-1}\right| \mathbf{F}\right\rangle\right\rangle-\gamma_{\infty}\left\langle\left\langle\mathbf{F} \cdot\left|(s-L)^{-1}\right| \mathbf{v}_{\text {c.m. }}\right\rangle\right\rangle
\end{aligned}
$$

using Eq. (8) and the defining equation for the operator $L^{+}$ [Eq. (9)]. When Eqs. (13) and (17) are substituted into Eq. (16), and like terms collected, we obtain

$$
\begin{aligned}
& \left\langle\left\langle\mathbf{F} \cdot\left|(s-L Q)^{-1}\right| \mathbf{F}\right\rangle\right\rangle \\
& \quad=\left\langle\left\langle\mathbf{F} \cdot\left|(s-L)^{-1}\right| \mathbf{F}\right\rangle\right\rangle /\left(1-\frac{1}{3 m N s k_{B} T}\left\langle\langle\mathbf{F} \cdot|(s-L)^{-1}\right.\right. \\
& \left.|\mathbf{F}\rangle\rangle-\frac{\gamma_{\infty}}{3 k_{B} T s}\left\langle\left\langle\mathbf{F} \cdot\left|(s-L)^{-1}\right| \mathbf{v}_{\text {c.m. }}\right\rangle\right\rangle\right) .
\end{aligned}
$$

The right-hand side of this equation is free of the operators $P$ and $Q$.

We now simplify Eq. (18) as follows: we introduce a function $\widetilde{C}(s)$, defined by

$$
\widetilde{C}(s) \equiv\left\langle\left\langle\mathbf{F} \cdot\left|(s-L)^{-1}\right| \mathbf{v}_{\text {c.m. }}\right\rangle\right\rangle .
$$

Laplace inverting this function, we obtain

$$
C(t)=\left\langle\left\langle\mathbf{F} \cdot\left|e^{t L}\right| \mathbf{v}_{\mathbf{c} . m} .\right\rangle\right\rangle
$$

which, on being differentiated with respect to time, produces

$\dot{C}(t)=\left\langle\left\langle\mathbf{F} \cdot\left|e^{t L} L\right| \mathbf{v}_{\mathrm{c} . \mathrm{m} .}\right\rangle\right\rangle=-\left\langle\left\langle\mathbf{F} \cdot\left|e^{t L}\right| \frac{1}{m N} \mathbf{F}+\gamma_{\infty} \mathbf{v}_{\mathrm{c} . \mathrm{m} .}\right\rangle\right\rangle$.

The Laplace transform of this equation, together with the result $C(0)=0$ leads to

$$
\widetilde{C}(s)=-\frac{1}{m N\left(s+\gamma_{\infty}\right)}\left\langle\left\langle\mathbf{F} \cdot\left|(s-L)^{-1}\right| \mathbf{F}\right\rangle\right\rangle .
$$

Substitution of this expression into Eq. (18) leads to the exact result

$$
\begin{aligned}
& \left\langle\left\langle\mathbf{F} \cdot\left|(s-L Q)^{-1}\right| \mathbf{F}\right\rangle\right\rangle \\
& \quad=\widetilde{F}(s) /\left[1-\widetilde{F}(s) /\left\{3 m N k_{B} T\left(s+\gamma_{\infty}\right)\right\}\right],
\end{aligned}
$$

where $\widetilde{F}(s) \equiv\left\langle\left\langle\mathbf{F} \cdot\left|(s-L)^{-1}\right| \mathbf{F}\right\rangle\right\rangle$. Using Eqs. (15) and (23) in (14), the self-diffusion coefficient can be finally written as

$$
\widetilde{D}(s)=D_{R} \frac{\gamma_{\infty}}{s+\gamma_{\infty}}\left[1-\frac{\widetilde{F}(s)}{3 m N k_{B} T\left(s+\gamma_{\infty}\right)}\right] .
$$

\section{APPROXIMATIONS}

So far the treatment has been exact, but we have merely rewritten the frequency-dependent self-diffusion coefficient in terms of another correlation function, the time-dependent autocorrelation function of the force. Although this function is unknown, it can at least be determined exactly to a certain order of approximation in the concentration $c$ of obstacles. The nature of this approximation is established as follows. By definition

$$
\langle\langle\mathbf{F} \cdot \mathbf{F}(t)\rangle\rangle=\sum_{i, j=1}^{N}\left\langle\left\langle\mathbf{F}_{i} \cdot \mathbf{F}_{j}(t)\right\rangle\right\rangle,
$$

where, as indicated earlier, $\mathbf{F}_{i}$ is the force acting on an individual chain segment. This force can be expressed in terms of the spatial derivative of the intermolecular potential $u(r)$ between monomer and obstacle, $r$ being the magnitude of the distance between the two. If there are $M$ obstacles in all, and $\mathbf{R}_{i}$ denotes the position of the $i$ th, then in Fourier space Eq. (25) can be rewritten as

$$
\begin{aligned}
\langle\langle\mathbf{F} \cdot \mathbf{F}(t)\rangle\rangle= & -\frac{1}{(2 \pi)^{6}} \sum_{i, j=1}^{N} \sum_{k, l=1}^{M} \int d \mathbf{q} \int d \mathbf{q}^{\prime} \hat{u}(\mathbf{q}) \hat{u}\left(\mathbf{q}^{\prime}\right) \\
& \times \mathbf{q} \cdot \mathbf{q}^{\prime}\left\langle\left\langle\exp \left[i \mathbf{q} \cdot\left(\mathbf{r}_{i}-\mathbf{R}_{k}\right)+i \mathbf{q}^{\prime} \cdot\left(\mathbf{r}_{j}(t)-\mathbf{R}_{l}\right)\right]\right\rangle\right\rangle .
\end{aligned}
$$

The average in Eq. (26) represents a four point correlation function that in general is difficult to treat without approximation. However, to first order in $c$, the cross correlations associated with the nondiagonal elements $k \neq l$ in the sum over scatterers can be neglected, as was done by Loring in his derivation of an expression for the equilibrium force cor- 
relation function. Within this approximation, and for a distribution of scatterers that is assumed to be uniform, as in Loring's calculations, one can reduce Eq. (26) to

$$
\langle\langle\mathbf{F} \cdot \mathbf{F}(t)\rangle\rangle=\frac{1}{(2 \pi)^{3}} c N \int d \mathbf{q} q^{2}|\hat{u}(\mathbf{q})|^{2} g(\mathbf{q}, t),
$$

where $g(\mathbf{q}, t)$, the dynamic structure factor of the Gaussian chain in the absence of scatterers, is defined $\mathrm{as}^{2}$

$$
g(\mathbf{q}, t)=\frac{1}{N} \sum_{i, j=1}^{N}\left\langle\exp \left[i \mathbf{q} \cdot\left(\mathbf{r}_{i}-\mathbf{r}_{j}(t)\right)\right]\right\rangle
$$

with the angular brackets now denoting an average over the conformational degrees of freedom of the polymer alone.

In principle, the calculation of $\langle\langle\mathbf{F} \cdot \mathbf{F}(t)\rangle\rangle$ can now be carried out rigorously to first order in the concentration of obstacles, because the expression for $g(\mathbf{q}, t)$ is known. However, this expression is fairly complicated; it is given by ${ }^{11}$

$g(\mathbf{q}, t)=\frac{1}{N} \exp \left(-D_{R} q^{2} t\right) \sum_{i, j=1}^{N} \exp \left(-q^{2} l^{2} \phi_{i j}(t) / 6\right)$,

where $l$ is the Kuhn length of the chain, and

$$
\begin{aligned}
\phi_{i j}(t)= & |i-j|+\frac{1}{N} \sum_{p=1}^{N-1} \frac{1}{\mu_{p}} \\
& \times \cos (p \pi i / N) \cos (p \pi j / N)\left[1-e^{-t^{\prime} \mu_{p}}\right]
\end{aligned}
$$

with $\mu_{p} \equiv \sin ^{2}(p \pi / 2 N)$ and $t^{\prime} \equiv 12 k_{B} T t / l^{2} \gamma_{\infty}$. The operations that lead from this expression to an expression for the center-of-mass displacement [by way of Eqs. (1) and (24)] include a summation over internal modes of the chain, an integral over wave vectors and an inverse Laplace transformation. Although individually these operations are not wholly intractable, in combination they have proved difficult to carry out. Even the approximation

$$
g(\mathbf{q}, t)=g(\mathbf{q}) \exp \left(-q^{2} D_{R} t\right),
$$

which is expected to provide a fairly sensible description of the dynamic structure factor in the limit of intermediate to long times, is not entirely successful in reducing the overall complexity of the problem.

To proceed further, therefore, we adopt a different strategy altogether. We assume first of all that the dynamic force correlation function has a simple exponential decay profile, i.e., we assume

$$
\begin{aligned}
\left\langle\left\langle\mathbf{F} \cdot\left|e^{t L}\right| \mathbf{F}\right\rangle\right\rangle & \equiv\langle\langle\mathbf{F} \cdot \mathbf{F}(t)\rangle\rangle \\
& \approx\langle\langle\mathbf{F} \cdot \mathbf{F}\rangle\rangle e^{-\mu t},
\end{aligned}
$$

where $1 / \mu$ can be interpreted as a characteristic decay constant that is as yet unknown. $\mu$ is given formally by the relation

$$
\mu=\langle\langle\mathbf{F} \cdot \mathbf{F}\rangle\rangle / \int_{0}^{\infty} d t\langle\langle\mathbf{F} \cdot \mathbf{F}(t)\rangle\rangle,
$$

which is obtained from Eqs. (32) and (33) by integrating over time from 0 to infinity, and then rearranging. Here, $\langle\langle\mathbf{F} \cdot \mathbf{F}\rangle\rangle$ is the equilibrium correlation function of the force. As described above, it has been determined approximately by Loring to first order in the concentration of the obstacles by assuming a uniform distribution for $P_{s}$, the scatterer distribution function. We shall regard $\langle\langle\mathbf{F} \cdot \mathbf{F}\rangle\rangle$ as being satisfactorily described by this expression, which is given by

$$
\langle\langle\mathbf{F} \cdot \mathbf{F}\rangle\rangle=(2 \pi)^{-3} c N \lambda,
$$

where $\lambda$ is the function

$$
\lambda=\int d \mathbf{q} q^{2} g(\mathbf{q})|\hat{u}(\mathbf{q})|^{2}
$$

and $g(\mathbf{q})$, the polymer structure factor in the absence of obstacles, is given by ${ }^{2}$

$$
g(\mathbf{q})=\frac{2 N}{q^{4} R_{g}^{2}}\left[\exp \left(-q^{2} R_{g}^{2}\right)+q^{2} R_{g}^{2}-1\right]
$$

with $R_{g}$ the radius of gyration of the chain.

If we now assume that the dynamic structure factor of the chain is adequately described by the simplified expression given in Eq. (31), the rate $\mu$ is given by

$$
\mu=D_{R} \lambda^{*},
$$

where $\lambda^{*}$ is defined as

$$
\lambda^{*}=\int d \mathbf{q} q^{2}|\hat{u}(\mathbf{q})|^{2} g(\mathbf{q}) / \int d \mathbf{q}|\hat{u}(\mathbf{q})|^{2} g(\mathbf{q}) .
$$

$\left\langle\left\langle\mathbf{r}_{\mathrm{c} . \mathrm{m} .}(t)^{2}\right\rangle\right\rangle$ itself is now readily determined to be

$$
\left\langle\left\langle\mathbf{r}_{\mathrm{c} . \mathrm{m} .}(t)^{2}\right\rangle\right\rangle=6 D_{R} t \Delta_{1}(t)\left[1-\eta \frac{\Delta_{2}(t)}{\Delta_{1}(t)}\right] .
$$

Here

$$
\Delta_{1}(t) \equiv 1-\frac{1}{\tau}\left(1-e^{-\tau}\right),
$$

where $\tau \equiv \gamma_{\infty} t$, and

$$
\begin{aligned}
\Delta_{2}(t) \equiv & \frac{1}{\sigma}\left[1-\frac{1}{\sigma \tau}\left(1-e^{-\sigma \tau}\right)\right]-\frac{2}{\sigma \tau}\left(1-e^{-\sigma \tau}\right) \\
& +\frac{2}{(\sigma-1) \tau}\left(e^{-\tau}-e^{-\sigma \tau}\right) \\
& +\frac{1}{\sigma-1}\left[e^{-\tau}-\frac{1}{(\sigma-1) \tau}\left(e^{-\tau}-e^{-\sigma \tau}\right)\right] .
\end{aligned}
$$

The parameter $\eta$ is the ratio of the concentration of obstacles $c$ to a characteristic concentration $c_{0}$ that we have identified with the combination of variables $24 \pi^{3} m k_{B} T \gamma_{\infty}^{2} / \lambda$, while the parameter $\sigma$ is the dimensionless combination of variables $D_{R} \lambda^{*} / \gamma_{\infty}$, which, from Eq. (38) is equivalent to $\mu / \gamma_{\infty} . \sigma$ is therefore, effectively, a ratio of friction coefficients, or of characteristic relaxation times. Since, in general, $1 / \mu \leqslant 1 / \gamma_{\infty}$ (the greater the degree of inhomogeneity in the medium, the faster, on average, the force on the chain at one instant decorrelates with the force at another), we expect that $\sigma \geqslant 1$.

As a final approximation, we introduce an ansatz that describes $\left\langle\left\langle\mathbf{r}_{\text {c.m. }}(t)^{2}\right\rangle\right\rangle$ in the region of high obstacle concentrations. This ansatz, which is in much the same spirit as the resummation approximation introduced in Ref. 6 to extend the range of lowest order perturbation theory, takes the form 


$$
\left\langle\left\langle\mathbf{r}_{\mathrm{c} . \mathrm{m} .}(t)^{2}\right\rangle\right\rangle q_{0}^{2}=\frac{6\left(D_{R} q_{0}^{2} / \gamma_{\infty}\right) \tau \Delta_{1}(t)}{1+\eta \Delta_{2}(t) / \Delta_{1}(t)}
$$

where $q_{0}$ is some characteristic wave vector corresponding to the range of the potential $u(r)$. In the limit $\eta \ll 1$, Eq. (43) clearly reduces to Eq. (40).

Other limits are of interest. For instance, at long times and low obstacle concentrations,

$$
\left\langle\left\langle\mathbf{r}_{\mathrm{c} . \mathrm{m} .}^{2}(t)\right\rangle\right\rangle \sim D_{R} t,
$$

where $D_{R} \sim 1 / N$. This is the classic Rouse limit, ${ }^{12}$ where the effects of disorder are sufficiently weak that the motion of the chain is essentially unhindered. Similarly, at long times [when $\Delta_{1}(t) \rightarrow 1$ and $\left.\Delta_{2}(t) \rightarrow 1 / \sigma\right]$ and at high obstacle concentrations,

$$
\left\langle\left\langle\mathbf{r}_{\mathrm{c} . \mathrm{m} .}^{2}(t)\right\rangle\right\rangle \sim D t,
$$

where $D$ is an effective diffusion constant that is proportional to $m k_{B} T \gamma_{\infty}^{2} D_{R} \sigma / c \lambda$. The molecular weight dependence of $D$ is expected to differ from $D_{R}$ on account of the factors $\sigma$ and $\lambda$ that appear in its definition. The additional $N$ dependence from these factors may be derived by appeal to the kinds of physical arguments used by Loring in his calculations, but a somewhat less qualitative derivation is possible if it is assumed that the interaction potential $u(r)$ between monomer and obstacle is a delta function, $u_{0} \delta(r)$, where $u_{0}$ is a measure of the strength of the interaction. With this choice of potential, $\hat{u}(\mathbf{q})$ becomes $u_{0}$, and the integral over wave vectors in the expressions for $\lambda$ and $\lambda^{*}$ [Eqs. (36) and (39)] is easily carried out, provided an ultraviolet cutoff $q_{0}$ [which effectively describes the range of the potential $u(r)$ or equivalently the radial dimensions of the obstacles] is introduced in the integral. In this way, $\lambda$ is determined to be

$$
\lambda=\frac{8 \pi N u_{0}^{2}}{R_{g}^{5}}\left[\frac{\sqrt{\pi}}{2} \Phi\left(q_{0} R_{g}\right)-q_{0} R_{g}+\frac{1}{3} q_{0}^{3} R_{g}^{3}\right],
$$

where $\Phi(x)$ is the error function. Similarly, $\lambda *$ is calculated as

$$
\begin{aligned}
\lambda^{*}= & \frac{1}{R_{g}^{2}}\left[\frac{\sqrt{\pi}}{2} \Phi\left(q_{0} R_{g}\right)-q_{0} R_{g}+\frac{1}{3} q_{0}^{3} R_{g}^{3}\right] /[\{1 \\
& \left.\left.-\exp \left(-q_{0}^{2} R_{g}^{2}\right)\right\} / q_{0} R_{g}-\sqrt{\pi} \Phi\left(q_{0} R_{g}\right)+q_{0} R_{g}\right] .
\end{aligned}
$$

The $N$ dependence of $\lambda$ and $\lambda *$ is readily extracted from these expressions in the limits $q_{0} R_{g} \gg 1$ (small scatterer limit) and $q_{0} R_{g} \ll 1$ (large scatterer limit). We find, first of all, that $\lambda^{*}$ is independent of $N$ in both these limits, whereas $\lambda$ is independent of $N$ in the small scatterer limit, and varies linearly with $N$ in the large scatterer limit. These results imply that $D \sim 1 / N^{2}$ if the scatterers are small in comparison to the average chain dimensions, and that $D \sim 1 / N^{3}$ if the scatterers are large in comparison to the average chain dimensions. These scaling results are in complete agreement with the corresponding results determined in Ref. 6.

Figures 1 and 2 show the variation of $\left\langle\left\langle\mathbf{r}_{\mathrm{c} . \mathrm{m} .}^{2}(t)\right\rangle\right\rangle q_{0}^{2}$ with $\tau \equiv \gamma_{\infty} t$ for two different values of the parameter $\sigma$ $\equiv D_{R} \lambda * / \gamma_{\infty}$ (10 and 1.01, respectively) and for various val-

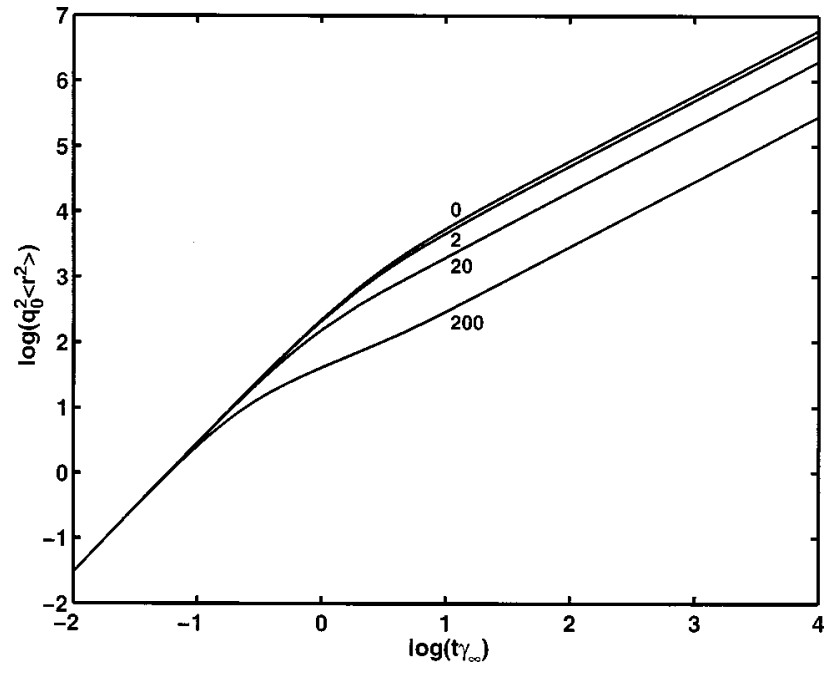

FIG. 1. The dimensionless mean square displacement of the center of mass of a Gaussian chain $\left\langle\left\langle\mathbf{r}_{\text {c.m. }}^{2}(t)\right\rangle\right\rangle q_{0}^{2}$, as calculated from Eq. (43), as a function of the reduced time $\gamma_{\infty} t$ at the indicated reduced densities $c / c_{0}$ of the obstacles for a fixed value 10 of the dimensionless parameter $\sigma$. The wave vector $q_{0}$ is a measure of the range of the segment-scatterer potential, $\gamma_{\infty}$ is the monomer friction coefficient and $c_{0}$ is a characteristic obstacle concentration. $c_{0}$ and $\sigma$ are defined after Eq. (42). In deriving these curves, the dimensionless variable $D_{R} q_{0}^{2} / \gamma_{\infty}$ in Eq. (43) has been assigned the value 100. The qualitative features of the curves are not sensitive to this value.

ues of the reduced concentration $\eta$. The curves show regions of linear time dependence at short and long times and a crossover region of anomalous (sublinear) time dependence at intermediate times whose width is a function of the amount of disorder in the medium and whose slope is a function of the value of the parameter $\sigma$ : the greater the amount of disorder in the medium, the greater the extent of this region, and the larger the value of $\sigma$, the stronger its dependence on $t$.

\section{DISCUSSION}

The present work is a re-examination of the problem of polymer diffusion in a random array of obstacles. The prob-

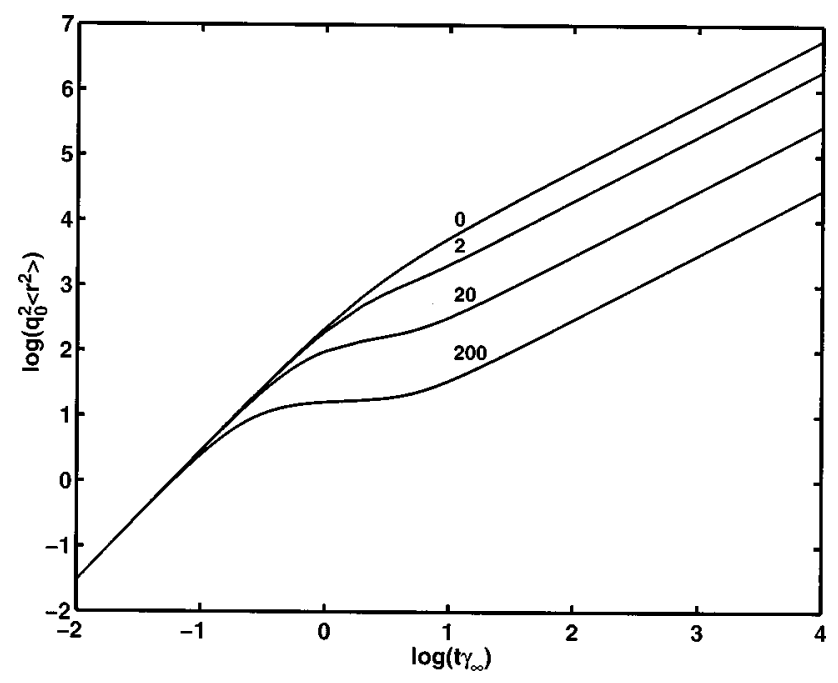

FIG. 2. $\left\langle\left\langle\mathbf{r}_{\text {c.m. }}^{2}(t)\right\rangle\right\rangle q_{0}^{2}$ vs $\gamma_{\infty} t$ for $\sigma=1.01$, all other parameters being identical to those in Fig. 1. 
lem has often been understood in terms of a phenomenological model in which chain diffusion takes place within the confines of an imaginary tube, the tube representing the coarse-grained effects of disorder in the medium. The motion of the chain in the tube is distinctively reptative, which is to say that it is directed largely along the contour of the polymer backbone. When a chain moves in this fashion, the displacement of its center of mass can be shown to vary as the square root of time in the limit of long times. Reptation is therefore diffusive in this limit, and can be characterized by an effective diffusion coefficient $D . D$ depends on the size of the chain $N$, and if $N$ is long enough, $D \sim N^{-2}$. This inverse quadratic dependence of $D$ on $N$ is a characteristic scaling prediction of the reptation model.

Loring's analytical treatment of polymer diffusion in random media is considerably more sophisticated than the reptation model, but its predictions are largely the same. In particular, it predicts the existence of a transition from Rouse to reptation dynamics at a certain concentration of obstacles, and it also predicts the existence of a long-time diffusive regime at high obstacle concentrations in which $D \sim N^{-a}$, where $2 \leqslant a \leqslant 3$. The precise value of the exponent $a$ depends on the relative sizes of polymer and obstacle: $a$ is 2 if the polymer is much larger than the obstacle, and $a$ is 3 if the polymer is much smaller than the obstacle. Although these results can be seen as providing a rigorous, microscopic basis for the reptation mechanism, they are obtained only if it is assumed that the friction coefficients parallel and perpendicular to the chain contour are distinct. Chain dynamics in Loring's approach is thus governed by a prior directional bias that may well predispose the polymer to diffuse reptatively.

No such bias is directly introduced into the present calculations, which adhere to the general methodology of Loring's projection operator formalism but eschew the steps that lead to the decoupling of parallel and perpendicular modes of motion by working with an exact relation between a generalized friction coefficient and the time correlation function of the total force on the chain. The absence of any projected dynamics in this relation suggests that one may be able to treat it accurately by means of molecular dynamics simulations. As we have shown, however, an analytic treatment is also possible if it is assumed that the dynamic force correlation function decays exponentially and that the decay constant can be calculated using a simplified representation of the dynamic structure factor of the free Gaussian chain. The results obtained with this approach are in complete qualitative agreement with the results of Ref. 6. In particular, $D$ is found to scale as $N^{-a}$, with $a$ either 2 or 3 depending on whether the polymer is much larger or much smaller than the obstacle. The variation of $\left\langle\left\langle\mathbf{r}_{\mathrm{c} . \mathrm{m} .}^{2}(t)\right\rangle\right\rangle$ with $t$ also shows the same three-regime behavior as the related curves in Ref. 6.

These general trends are also seen in the curves obtained in the simulations of Baumgartner and Muthukumar, ${ }^{7-9}$ which looked at the dynamics of polymers with and without excluded volume in a porous solid. The solid was modelled as a three-dimensional cubic lattice with a random distribution of hard cubes, while the polymer was modelled either as a freely jointed chain or as a necklace of hard spheres joined by rigid links. (Simulations in two dimensions were also reported, but these will not be discussed here.) A single Gaussian chain of given $N$ in a solid of porosity $p$ is found to behave diffusively at short and long times and anomalously at intermediate times, just as in the calculations discussed here and in Ref. 6. The short time diffusion coefficient $D$ determined in the simulations is inversely proportional to $N$, but the long time diffusion coefficient varies as $N^{-\alpha}$, where $\alpha$ is $p$ dependent. At porosities above the percolation threshold of $0.6883, \alpha$ varies roughly between 1 and 2, while at porosities below $0.6883, \alpha$ varies roughly between 2 and 3 . However, at these latter values of $p$, the variation of $D$ with $N$ is not unambiguously a power law; it is equally well described by $D \sim N^{-1} \exp (-B N), B$ being a constant. ${ }^{9}$ These various results can be reconciled with Loring's and our analytical calculations in the small and large obstacle limit, but the degree of support for a reptative mode of motion is not necessarily high, given the range of possible behaviors for the variation of $D$ with $N$. (Indeed, in Ref. 7, the authors go so far as to state that reptation is absent.) Differences between the simulation and reptation results at low porosity have been ascribed to chain collapse and to the existence of entropic bottlenecks in the solid.

When excluded volume interactions are incorporated into the simulations by using the pearl necklace model for the chain, the variation of $\left\langle\left\langle\mathbf{r}_{\mathrm{c} . \mathrm{m} .}^{2}(t)\right\rangle\right\rangle$ with $t$ also exhibits three regimes of behavior: a short and long time diffusive regime and an intermediate time anomalous regime. As with the Gaussian chain, at short times $D \sim N^{-1}$ for all $p$, but at longer times $D$ is not unambiguously a power law in $N$, and if it is described by a power law, the exponent is not necessarily -2 . The self-avoiding walk simulations differ in one respect from the Gaussian chain simulations: chain collapse is not observed to occur.

There is one case in which the simulations produce the result $D \sim N^{-2}$ at long times. This is the case of the random or self-avoiding walk in a periodic array of infinitely long hard rods, and here reptation can almost certainly be implicated in the dynamics of the chain.

In summary, then, we have shown that a microscopic model of chain dynamics in a random array of obstacles leads to results that are largely consistent with the results of simulations and with the predictions of reptation. The fact that this is so despite the absence in our calculations of any obvious discrimination between parallel and perpendicular modes of segmental motion perhaps suggests ${ }^{13}$ (when considered along side the somewhat equivocal results of simulations) that at least one of the characteristic scaling predictions of reptation, viz., $D \sim N^{-2}$, is not a stringent enough test of whether reptation does in fact occur.

\section{ACKNOWLEDGMENTS}

We are grateful to Professor Roger Loring, Cornell University, for helpful comments and discussions.

${ }^{1}$ P.-G. de Gennes, Scaling Concepts in Polymer Physics (Cornell, Ithaca, 1979).

${ }^{2}$ M. Doi and S. F. Edwards, The Theory of Polymer Dynamics (Oxford, New York, 1986). 
${ }^{3}$ M. Bishop, D. Ceperley, H. L. Frisch, and M. H. Kalos, J. Chem. Phys. 76, 1557 (1982)

${ }^{4}$ K. E. Evans and S. F. Edwards, J. Chem. Soc., Faraday Trans. 77, 1891,1929 (1981)

${ }^{5}$ A. Kolinski, J. Skolnick, and R. Yaris, J. Chem. Phys. 86, 1567 (1987).

${ }^{6}$ R. F. Loring, J. Chem. Phys. 88, 6631 (1988).

${ }^{7}$ A. Baumgartner and M. Muthukumar, J. Chem. Phys. 87, 3082 (1987).
${ }^{8}$ M. Muthukumar and A. Baumgartner, Macromolecules 22, 1937,1941 (1989).

${ }^{9}$ A. Baumgartner and M. Muthukumar, Adv. Chem. Phys. 94, 625 (1996).

${ }^{10}$ W. Hess, Macromolecules 19, 1395 (1986); 20, 2587 (1987).

${ }^{11}$ A. Z. Akcasu, M. Benmouna, and C. C. Han, Polymer 21, 866 (1980).

${ }^{12}$ P. E. Rouse, J. Chem. Phys. 21, 1272 (1953).

${ }^{13}$ J. Skolnick and A. Kolinski, Adv. Chem. Phys. 78, 223 (1990). 